\title{
The Structure of $\mathbb{Z}_{2} \mathbb{Z}_{2^{s}}$-Additive Codes: Bounds on the Minimum Distance
}

\author{
Ismail Aydogdu and Irfan Siap* \\ Department of Mathematics,Faculty of Arts and Science, Yildiz Technical University,Istanbul,Turkey
}

Received: 8 Mar. 2013, Revised: 11 Jul. 2013, Accepted: 12 Jul. 2013

Published online: 1 Nov. 2013

\begin{abstract}
Z}_{2} \mathbb{Z}_{4}$-additive codes, as a special class of abelian codes, have found a very welcoming place in the recent studies of algebraic coding theory. This family in one hand is similar to binary codes on the other hand is similar to quaternary codes. The structure of $\mathbb{Z}_{2} \mathbb{Z}_{4}$ additive codes and their duals has been determined lately. In this study we investigate the algebraic structure of $\mathbb{Z}_{2} \mathbb{Z}_{2^{s}}$-additive codes which are a natural generalization of $\mathbb{Z}_{2} \mathbb{Z}_{4}$-additive codes. We present the standard form of the generator and parity-check matrices of the $\mathbb{Z}_{2} \mathbb{Z}_{2^{s}}$-additive codes. Also, we give two bounds on the minimum distance of $\mathbb{Z}_{2} \mathbb{Z}_{2^{s}}$-additive codes and compare them.
\end{abstract}

Keywords: Additive Codes, $\mathbb{Z}_{2} \mathbb{Z}_{4}$-Linear Codes, $\mathbb{Z}_{2} \mathbb{Z}_{2^{s}}$-Additive Codes.

\section{Introduction}

Let $\mathbb{Z}_{2}$ and $\mathbb{Z}_{4}$ be the ring of integers modulo 2 and 4 , respectively. We denote the set of all binary vectors of length $n$ by $\mathbb{Z}_{2}^{n}$ and the set of all $n$-tuples over the ring $\mathbb{Z}_{4}$ by $\mathbb{Z}_{4}^{n}$. Any nonempty subset $C$ of $\mathbb{Z}_{2}^{n}$ is a binary code and a subgroup of $\mathbb{Z}_{2}^{n}$ is called a binary linear code or a $\mathbb{Z}_{2}$ - linear code. Equivalently, any nonempty subset $\mathscr{C}$ of $\mathbb{Z}_{4}^{n}$ is a quaternary code and a subgroup of $\mathbb{Z}_{4}^{n}$ is called a quaternary linear code.

We can view binary codes as quaternary codes under the Gray map defined in [8] as $\phi_{1}(0)=(0,0) \phi_{1}(1)=$ $(0,1), \phi_{1}(2)=(1,1), \phi_{1}(3)=(1,0)$. If $\mathscr{C}$ is a quaternary linear code then the binary code $C=\phi_{1}(\mathscr{C})$ is said to be a $\mathbb{Z}_{4}$ - linear code.

Additive codes were first defined by Delsarte in 1973 in terms of association schemes $[5,6]$. In a translation association scheme, an additive code is generally defined as a subgroup of the underlying abelian group. On the other hand, translation invariant propelinear codes were first defined in 1997 and it was proved that all these binary codes are isomorphic to subgroups of $\mathbb{Z}_{2}^{\alpha} \times \mathbb{Z}_{4}^{\beta} \times \mathbb{Q}_{8}^{\sigma}$ where $\mathbb{Q}_{8}$ is the nonabelian quaternion group with eight elements [9]. If the association scheme is the binary Hamming scheme, that is, when the underlying abelian group is of order $2^{n}$, the additive codes coincide with the abelian translation invariant propelinear codes. Therefore, the only structures for the abelian group are those of the form $\mathbb{Z}_{2}^{\alpha} \times \mathbb{Z}_{4}^{\beta}$, where $\alpha+2 \beta=n$ [9]. Hence, the subgroups $\mathscr{C}$ of $\mathbb{Z}_{2}^{\alpha} \times \mathbb{Z}_{4}^{\beta}$ are the only additive codes in the binary Hamming scheme.

Most of the concepts on $\mathbb{Z}_{2} \mathbb{Z}_{4}$-additive codes have been described in [2]. In this paper, we study $\mathbb{Z}_{2} \mathbb{Z}_{2^{s}}$-additive codes for $s>1$, where $\mathbb{Z}_{2} \mathbb{Z}_{4}$-additive codes are a special case. First, we give the definition of $\mathbb{Z}_{2} \mathbb{Z}_{2^{s}}$-additive codes and find some fundamental parameters. Next, we give the standard form of generator and parity-check matrices for $\mathbb{Z}_{2} \mathbb{Z}_{2^{s}}$-additive codes and we show some examples of these codes. Finally, we investigate two bounds on the minimum distance of $\mathbb{Z}_{2} \mathbb{Z}_{2^{s}}$-additive codes, compare them and deduce some results.

\section{Preliminaries}

An extended Gray map for $\mathbb{Z}_{2} \mathbb{Z}_{4}$ additive codes is defined by Borges et al. in [2] as follows: $\Phi_{1}: \mathbb{Z}_{2}^{\alpha} \times \mathbb{Z}_{4}^{\beta} \longrightarrow \mathbb{Z}_{2}^{n}$, where $n=\alpha+2 \beta$, given by for all $x \in \mathbb{Z}_{2}^{\alpha}$ and $y=\left(y_{1}, y_{2}, \ldots, y_{\beta}\right) \in \mathbb{Z}_{4}^{\beta}$

$$
\Phi_{1}(x, y)=\left(x, \phi_{1}\left(y_{1}\right), \ldots, \phi_{1}\left(y_{\beta}\right)\right)
$$

\footnotetext{
*Corresponding author e-mail: isiap@yildiz.edu.tr
} 
where $\phi_{1}: \mathbb{Z}_{4} \longrightarrow \mathbb{Z}_{2}^{2}$ is the usual Gray map that is;

$\phi_{1}(0)=(0,0), \phi_{1}(1)=(0,1), \phi_{1}(2)=(1,1), \phi_{1}(3)=(1,0)$.

This Gray map is an isometry which transforms Lee distances defined in a $\mathbb{Z}_{2} \mathbb{Z}_{4}$-additive code over $\mathbb{Z}_{2}^{\alpha} \times \mathbb{Z}_{4}^{\beta}$ to Hamming distances defined in the binary code $C=\Phi_{1}(\mathscr{C})$. Hence, the length of the binary code $C$ is $n=\alpha+2 \beta$.

We know that if $\mathscr{C}$ is a $\mathbb{Z}_{2} \mathbb{Z}_{4}$-additive code then it is a subgroup of $\mathbb{Z}_{2}^{\alpha} \times \mathbb{Z}_{4}^{\beta}$ so, it is also isomorphic to an abelian structure $\mathbb{Z}_{2}^{\gamma} \times \mathbb{Z}_{4}^{\delta}$. Therefore, $\mathscr{C}$ is of type $2^{\gamma} 4^{\delta}$ as a group and it has $|\mathscr{C}|=2^{\gamma+2 \delta}$ codewords. Let $X$ (respectively $Y$ ) be the set of $\mathbb{Z}_{2}$ (respectively $\mathbb{Z}_{4}$ ) coordinate positions, so $|X|=\alpha$ and $|Y|=\beta$. Call $\mathscr{C}_{X}$ (respectively $\mathscr{C}_{Y}$ ) the punctured code of $\mathscr{C}$ by deleting the coordinates outside $X$ (respectively $Y$ ). Let $\mathscr{C}_{b}$ the subcode of $\mathscr{C}$ which contains all order two codewords and let $\kappa$ be the dimension of $\left(\mathscr{C}_{b}\right)_{X}$, which is a binary linear code. Considering all these parameters, $\mathscr{C}$ is called a $\mathbb{Z}_{2} \mathbb{Z}_{4}$-additive code of type $(\alpha, \beta ; \gamma, \delta ; \kappa)$. We say that the binary image $C=\phi_{1}(\mathscr{C})$ is a $\mathbb{Z}_{2} \mathbb{Z}_{4}$-linear code of length $n=\alpha+2 \beta$ [2].

It is shown in [2] that the generator and parity-check matrices for a $\mathbb{Z}_{2} \mathbb{Z}_{4}$-additive code $\mathscr{C}$ of type $(\alpha, \beta ; \gamma, \delta ; \kappa)$ can be written in the following standard forms;

$$
\begin{gathered}
G_{1}=\left[\begin{array}{ccccc}
I_{\kappa} & T_{b} & 2 T_{2} & 0 & 0 \\
0 & 0 & 2 T_{1} & 2 I_{\gamma-\kappa} & 0 \\
0 & S_{b} & S_{q} & R & I_{\delta}
\end{array}\right] \\
H_{1}=\left[\begin{array}{ccccc}
T_{b}^{t} & I_{\alpha-\kappa} & 0 & 0 & 2 S_{b}^{t} \\
0 & 0 & 0 & 2 I_{\gamma-\kappa} & 2 R^{t} \\
T_{2}^{t} & 0 & I_{\beta+\kappa-\gamma-\delta} & T_{1}^{t} & -\left(S_{q}+R T_{1}\right)^{t}
\end{array}\right]
\end{gathered}
$$

where $T_{1}, T_{2}, T_{b}, S_{b}$ and $R$ are matrices over $\mathbb{Z}_{2}$ and $S_{q}$ is a matrix over $\mathbb{Z}_{4}$.

We define $\mathbb{Z}_{2} \mathbb{Z}_{2^{s}}$-additive codes which are a natural generalization of $\mathbb{Z}_{2} \mathbb{Z}_{4}$-additive codes as follows.

Definition 2.1. For a positive integer $s>1$, the code $\mathscr{C}$

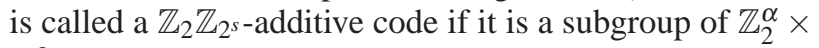
$\mathbb{Z}_{2^{s}}^{\beta}$. We say the binary image $\Phi(\mathscr{C})=C$ is a $\mathbb{Z}_{2} \mathbb{Z}_{2^{s}}$-linear code of length $n=\alpha+2^{s-1} \beta$ where $\Phi$ is Gray map from $\mathbb{Z}_{2}^{\alpha} \times \mathbb{Z}_{2^{s}}^{\beta}$ to $\mathbb{Z}_{2}^{n}$ defined by

$$
\Phi(x, y)=\left(x_{1}, \ldots, x_{\alpha}, \phi\left(y_{1}\right), \ldots, \phi\left(y_{\beta}\right)\right)
$$

for all $x=\left(x_{1}, x_{2}, \ldots, x_{\alpha}\right) \in \mathbb{Z}_{2}^{\alpha}, y=\left(y_{1}, y_{2}, \ldots, y_{\beta}\right) \in \mathbb{Z}_{2^{s}}^{\beta}$ and $\phi: \mathbb{Z}_{2^{s}} \longrightarrow \mathbb{Z}_{2}^{2^{s-1}}$ is generalization of the usual Gray map defined in [4].

In view of the definition, we can say that a $\mathbb{Z}_{2} \mathbb{Z}_{2^{s}}$-additive code is isomorphic to an abelian structure $\mathbb{Z}_{2}^{k_{0}+k_{s}} \times \mathbb{Z}_{2^{s}}^{k_{1}} \times \ldots \times \mathbb{Z}_{4}^{k_{s-1}}$. Here, the first $\alpha$ coordinates of codewords of $\mathscr{C}$ are elements from $\mathbb{Z}_{2}$ and the remaining $\beta$ coordinates are from $\mathbb{Z}_{2^{s}}$. Under these parameters we say that $\mathscr{C}$ is of type $\left(\alpha, \beta ; k_{0}, k_{1}, k_{2}, \ldots, k_{s}\right)$.

\section{Generator matrices of $\mathbb{Z}_{2} \mathbb{Z}_{2^{s}}$-additive codes}

Theorem 3.1. Let $\mathscr{C}$ be a $\mathbb{Z}_{2} \mathbb{Z}_{2^{s}}$-additive code of type $\left(\alpha, \beta ; k_{0}, k_{1}, k_{2}, \ldots, k_{s}\right)$. Then $\mathscr{C}$ is permutation equivalent to a $\mathbb{Z}_{2} \mathbb{Z}_{2^{s}}$-additive code with the standard form matrix,

$$
G_{S}=\left[\begin{array}{cc}
\bar{A} & T \\
S & A
\end{array}\right]
$$

where

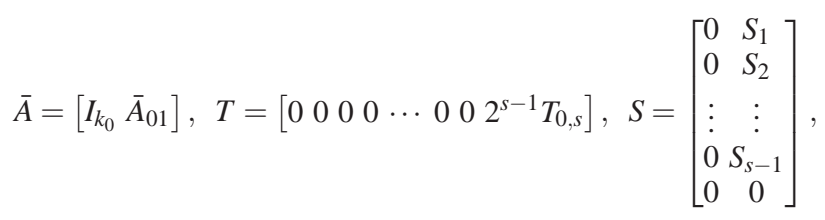

and

$A=\left[\begin{array}{ccccccc}I_{k_{1}} & A_{01} & A_{02} & \cdots & A_{0, s-2} & A_{0, s-1} & A_{0, s} \\ 0 & 2 I_{k_{2}} & 2 A_{12} & \cdots & 2 A_{1, s-2} & 2 A_{1, s-1} & 2 A_{1, s} \\ \vdots & \vdots & \vdots & \vdots & \vdots & \vdots & \vdots \\ 0 & 0 & 0 & \cdots & 2^{s-2} I_{k_{s-1}} & 2^{s-2} A_{s-2, s-1} & 2^{s-2} A_{s-2, s} \\ 0 & 0 & 0 & \cdots & 0 & 2^{s-1} I_{k_{s}} & 2^{s-1} A_{s-1, s}\end{array}\right]$.

Here, $\bar{A}_{01}$ is a matrix over $\mathbb{Z}_{2}$ and $A_{k l}$ are matrices over $\mathbb{Z}_{2^{s}}$ for $0 \leq k<l \leq s$. And $I_{k_{m}}$ are identity matrices of order $k_{m}$ for $1 \leq m \in \mathbb{Z}$.

For $1 \leq i \leq s-1, S_{i}$ are matrices over $\mathbb{Z}_{2}$ and $T_{0, s}$ is a matrix over $\mathbb{Z}_{2^{s}}$. Note that $\mathscr{C}$ has $|\mathscr{C}|=2^{k_{0}} 2^{s k_{1}} 2^{(s-1) k_{2}} \ldots 2^{k_{s}}$ codewords.

Proof. We prove the theorem in four steps for the $\mathbb{Z}_{2} \mathbb{Z}_{2^{s}}$-additive code $\mathscr{C}$ :

Step 1: Let $\mathscr{C}_{1}$ consist of the elements of $\mathscr{C}$ of order 2 where at least one entry in the first $\alpha$ coordinates is nonzero. And let $\mathscr{C}_{0}=\mathscr{C} \backslash \mathscr{C}_{1}$. Then it is clear that $\mathscr{C}=\mathscr{C}_{0} \cup \mathscr{C}_{1}$ and $\mathscr{C}_{0} \cap \mathscr{C}_{1}=\varnothing$. Let $\mathscr{C}_{1}$ be a subgroup of order $k_{0}$ then, after necessary permutations, we have the generator matrix of $\mathscr{C}$ in the form:

$$
\left[\begin{array}{ccc}
I_{k_{0}} & \bar{A}_{01} & 2^{s-1} D \\
\vdots & \vdots & \vdots \\
\vdots & \vdots & \vdots
\end{array}\right] \text {, where } \bar{A}_{01} \text { is a matrix over } \mathbb{Z}_{2} \text { and }
$$

$D$ is a matrix over $\mathbb{Z}_{2^{s}}$. 
Step 2: We assume that the first $k_{0}$ elements that generate $\mathscr{C}_{0}$ are zero then by applying necessary operations, that is;

$$
\left[\begin{array}{ccc}
I_{k_{0}} & \bar{A}_{01} & 2^{s-1} D \\
0 & \vdots & \vdots \\
0 & \vdots & \vdots
\end{array}\right]
$$

Step 3: Let $\mathscr{C}_{0}^{R}$ be a subgroup of $\mathbb{Z}_{2^{s}}$ obtained by taking the last $\beta$ coordinates of the elements in $\mathscr{C}_{0}$, i.e., a restriction of $\mathscr{C}_{0}$ in the last $\beta$ positions. Since $\mathscr{C}_{0}^{R}$ is a subgroup of $\mathbb{Z}_{2^{s}}$, we know from [3] that $\mathscr{C}_{0}^{R}$ is generated by a matrix of the form;

$A=\left[\begin{array}{ccccccc}I_{k_{1}} & A_{01} & A_{02} & \cdots & A_{0, s-2} & A_{0, s-1} & A_{0, s} \\ 0 & 2 I_{k_{2}} & 2 A_{12} & \cdots & 2 A_{1, s-2} & 2 A_{1, s-1} & 2 A_{1, s} \\ \vdots & \vdots & \vdots & \vdots & \vdots & \vdots & \vdots \\ 0 & 0 & 0 & \cdots & 2^{s-2} I_{k_{s-1}} & 2^{s-2} A_{s-2, s-1} & 2^{s-2} A_{s-2, s} \\ 0 & 0 & 0 & \cdots & 0 & 2^{s-1} I_{k_{s}} & 2^{s-1} A_{s-1, s}\end{array}\right]$.

So, the generator matrix of $\mathscr{C}$ has the form

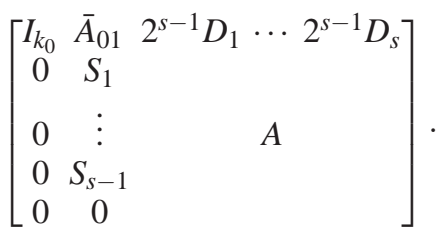

Step 4: Let us denote $\left[2^{s-1} D_{1} \cdots 2^{s-1} D_{s}\right]$ by $G_{0}$. By applying necessary row operations, we can make the first $k_{1}$ entries of $G_{0}$, which is the submatrix $2^{s-1} D_{1}$ to be zero. Similarly, $2^{s-1} D_{2}, \ldots, 2^{s-1} D_{s-1}$ can be made zero. It is clear that $S_{1}, \ldots, S_{s-1}$ are matrices over $\mathbb{Z}_{2}$. Hence, we have the result.

Now, we state a special case of Theorem 3.1 which gives the results in [2] by just applying permutations.

Corollary 3.2. If $\mathscr{C}_{1}$ is a $\mathbb{Z}_{2} \mathbb{Z}_{4}$-additive code of type $\left(\alpha, \beta ; k_{0}, k_{1}, k_{2}\right)$ then the generator matrix of $\mathscr{C}_{1}$ is in the form of;

$$
G=\left[\begin{array}{ccccc}
I_{k_{0}} & \bar{A}_{01} & 0 & 0 & 2 T_{02} \\
0 & S_{1} & I_{k_{1}} & A_{01} & A_{02} \\
0 & 0 & 0 & 2 I_{k_{2}} & 2 A_{12}
\end{array}\right]
$$

Note that this matrix is permutation equivalent to matrix of the form (1). Next, we present examples that illustrate Theorem 3.1.

Example 3.3. Let $\mathscr{C}_{2}$ be a $\mathbb{Z}_{2} \mathbb{Z}_{8}$-additive code of type $\left(\alpha, \beta ; k_{0}, k_{1}, k_{2}, k_{3}\right)$ with $s=3$. Applying Theorem 3.1, $\mathscr{C}_{2}$ is permutation equivalent to a $\mathbb{Z}_{2} \mathbb{Z}_{8}$-additive code with generator matrix of the form,

$$
G_{2}=\left[\begin{array}{cccccc}
I_{k_{0}} & \bar{A}_{01} & 0 & 0 & 0 & 4 T_{03} \\
0 & S_{1} & I_{k_{1}} & A_{01} & A_{02} & A_{03} \\
0 & S_{2} & 0 & 2 I_{k_{2}} & 2 A_{12} & 2 A_{13} \\
0 & 0 & 0 & 0 & 4 I_{k_{3}} & 4 A_{23}
\end{array}\right] .
$$

Example 3.4. Let $\mathscr{C}_{3}$ be a $\mathbb{Z}_{2} \mathbb{Z}_{8}$-additive code of type $(3,3 ; 1,3,0,0)$ with generator matrix

$$
\left[\begin{array}{llllll}
1 & 1 & 0 & 2 & 4 & 6 \\
0 & 1 & 1 & 0 & 4 & 5 \\
0 & 0 & 1 & 1 & 3 & 7 \\
1 & 0 & 1 & 0 & 5 & 4
\end{array}\right]
$$

then, the standard form of this generator matrix is as follows:

$$
\left[\begin{array}{llllll}
1 & 1 & 0 & 0 & 0 & 0 \\
0 & 0 & 1 & 1 & 0 & 0 \\
0 & 1 & 1 & 0 & 1 & 0 \\
0 & 1 & 1 & 0 & 0 & 1
\end{array}\right]
$$

\section{Duality of $\mathbb{Z}_{2} \mathbb{Z}_{2^{s}-\text { additive codes }}$}

For linear codes over finite fields and finite rings, the well-known standard concept of orthogonality is used. However, in the case of $\mathbb{Z}_{2} \mathbb{Z}_{4}$ the concept of orthogonality is given differently [2]. Here, we generalize this orthogonality and study the duality concept accordingly. Specifically, in this section, we study the duality for $\mathbb{Z}_{2} \mathbb{Z}_{2^{s}}$-additive codes.

We introduce an inner product for two vectors $u, v \in$ $\mathbb{Z}_{2}^{\alpha} \times \mathbb{Z}_{2^{s}}^{\beta}$ to be defined as;

$$
\langle u, v\rangle=2^{s-1}\left(\sum_{i=1}^{\alpha} u_{i} v_{i}\right)+\sum_{j=\alpha+1}^{\alpha+\beta} u_{j} v_{j} \in \mathbb{Z}_{2^{s}} .
$$

Please note that the case $s=2$ coincides with the definition in [2].

Let $\mathscr{C}$ be a $\mathbb{Z}_{2} \mathbb{Z}_{2^{s}}$-additive code. The additive dual code of $\mathscr{C}$, denoted by $\mathscr{C}^{\perp}$, is defined as

$$
\mathscr{C}^{\perp}=\left\{v \in \mathbb{Z}_{2}^{\alpha} \times \mathbb{Z}_{2^{s}}^{\beta} \mid\langle u, v\rangle=0 \text { for all } u \in \mathscr{C}\right\} .
$$

It is easy to check that $\mathscr{C}^{\perp}$ is a subgroup of $\mathbb{Z}_{2}^{\alpha} \times \mathbb{Z}_{2^{s}}^{\beta}$,

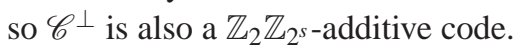

Now, we define the following maps for constructing

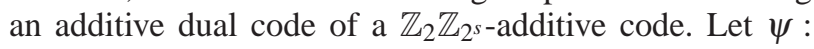
$\mathbb{Z}_{2^{s}} \longrightarrow \mathbb{Z}_{2}$ be the usual modulo 2 map, that is,

$$
\psi(x)=\left\{\begin{array}{l}
0, \quad \text { if } x=2 k \\
1, \text { if } x=2 k+1
\end{array}\right.
$$

for all $x \in \mathbb{Z}_{2^{s}}$ and $0 \leq k \in \mathbb{Z}$. Let $\imath: \mathbb{Z}_{2} \longrightarrow \mathbb{Z}_{2^{s}}$ be the identity map that is defined as $\imath(0)=0, \imath(1)=1$. Finally we define $\chi: \mathbb{Z}_{2} \longrightarrow \mathbb{Z}_{2^{s}}$, which is the usual inclusion from the additive structure in $\mathbb{Z}_{2}$ to $\mathbb{Z}_{2^{s}}$, that is, $\chi(0)=0$ and 
$\chi(1)=2^{s-1}$. We use the same notations for extensions of these functions. Hence we have, $\left(\psi, I_{d}\right): \mathbb{Z}_{2^{s}}^{\alpha} \times \mathbb{Z}_{2^{s}}^{\beta} \longrightarrow$ $\mathbb{Z}_{2}^{\alpha} \times \mathbb{Z}_{2^{s}}^{\beta},\left(l, I_{d}\right): \mathbb{Z}_{2}^{\alpha} \times \mathbb{Z}_{2^{s}}^{\beta} \longrightarrow \mathbb{Z}_{2^{s}}^{\alpha} \times \mathbb{Z}_{2^{s}}^{\beta}$, and $\left(\chi, I_{d}\right):$ $\mathbb{Z}_{2}^{\alpha} \times \mathbb{Z}_{2^{s}}^{\beta} \longrightarrow \mathbb{Z}_{2^{s}}^{\alpha} \times \mathbb{Z}_{2^{s}}^{\beta}$.

Lemma 4.1. Let $u \in \mathbb{Z}_{2}^{\alpha} \times \mathbb{Z}_{2^{s}}^{\beta}$ and $v \in \mathbb{Z}_{2^{s}}^{\alpha+\beta}$ then $\langle\chi(u), v\rangle_{2^{s}}=\langle u, \psi(v)\rangle$, where $\langle,\rangle_{2^{s}}$ denotes the standard inner product of vectors in $\mathbb{Z}_{2^{s}}$.

Proof. For $u \in \mathbb{Z}_{2}^{\alpha} \times \mathbb{Z}_{2^{s}}^{\beta}$ and $v \in \mathbb{Z}_{2^{s}}^{\alpha+\beta}$,

$$
\begin{aligned}
\langle\chi(u), v\rangle_{2^{s}} & =\sum_{i=1}^{\alpha}\left(2^{s-1} u_{i}\right) v_{i}+\sum_{j=\alpha+1}^{\alpha+\beta} u_{j} v_{j} \\
& =\sum_{i=1}^{\alpha}\left(2^{s-1} u_{i}\right)\left(v_{i} \bmod 2\right)+\sum_{j=\alpha+1}^{\alpha+\beta} u_{j} v_{j} \\
& =\langle u, \psi(v)\rangle .
\end{aligned}
$$

Corollary 4.2. If $u, v \in \mathbb{Z}_{2}^{\alpha} \times \mathbb{Z}_{2^{s}}^{\beta}$ then $\langle\chi(u), \imath(v)\rangle_{2^{s}}=\langle u, v\rangle$.

Proof. By Lemma 4.1,

$$
\langle\chi(u), \imath(v)\rangle_{2^{s}}=\langle u, \psi(l(v))\rangle=\langle u, v\rangle .
$$

Proposition 4.3. Let $\mathscr{C}$ be a $\mathbb{Z}_{2} \mathbb{Z}_{2^{s}}$-additive code of type $\left(\alpha, \beta ; k_{0}, k_{1}, \ldots, k_{s}\right)$. Then, $\mathscr{C}^{\perp}=\psi\left(\chi(\mathscr{C})^{\perp}\right)$.

Proof. Let $v \in \mathscr{C}^{\perp}$. So, for all $u \in \mathscr{C}$, we have $\langle u, v\rangle=0$. By Corollary $4.2\langle u, v\rangle=\langle\chi(u), \imath(v)\rangle_{2 s}$. Hence, $\psi(l(v))=v \in \psi\left(\chi(\mathscr{C})^{\perp}\right)$, and $\mathscr{C}^{\perp} \subseteq \psi\left(\chi(\mathscr{C})^{\perp}\right)$. On the other hand, if $v \in \chi(\mathscr{C})^{\perp}$, then $\langle\chi(u), v\rangle_{2^{s}}=0$, for all $u \in \mathscr{C}$. By Lemma 4.1, $\langle\chi(u), v\rangle_{2^{s}}=\langle u, \psi(v)\rangle=0$. Thus, $\psi\left(\chi(\mathscr{C})^{\perp}\right) \subseteq \mathscr{C}^{\perp}$

\section{Parity-check matrices of $\mathbb{Z}_{2} \mathbb{Z}_{2^{s}-\text { additive }}$ codes}

In this section we present the standard form of

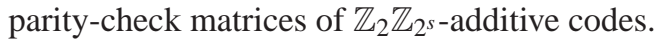

Let $k(A)$ be the number of rows of a matrix $A$. For $i=$ $1, \ldots, s$ we denote by $k_{i}(A)$ the number of rows of $A$ that are divisible by $2^{i-1}$ but not $2^{i}$. Hence $k(A)=\sum_{i=1}^{s} k_{i}(A)$.

Theorem 5.1. Let $\mathscr{C}$ be a $\mathbb{Z}_{2} \mathbb{Z}_{2^{s}}$-additive code of type $\left(\alpha, \beta ; k_{0}, k_{1}, k_{2}, \ldots, k_{s}\right)$ with standard form matrix (4). Then, the generator matrix of $\mathscr{C}^{\perp}$ is given by

$$
H_{S}=\left[\begin{array}{cc}
A_{1} & U \\
T_{1} & B
\end{array}\right]
$$

where

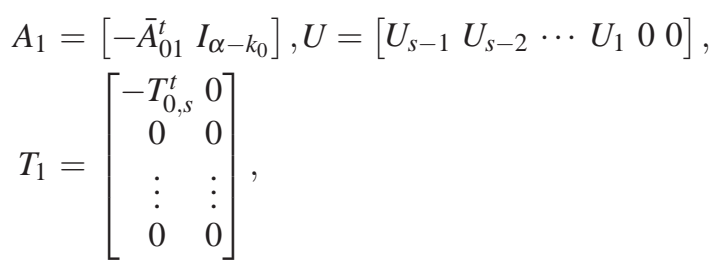

$$
B=\left[\begin{array}{cccccc}
B_{0, s} & B_{0, s-1} & \cdots & B_{0,2} & B_{0,1} & I_{\beta-k(A)} \\
2 B_{1, s} & 2 B_{1, s-1} & \cdots & 2 B_{1,2} & 2 I_{k_{s}(A)} & 0 \\
\vdots & \vdots & \vdots & \vdots & \vdots & \vdots \\
2^{s-1} B_{s-1, s} & 2^{s-1} I_{k_{2}(A)} & \cdots & 0 & 0 & 0
\end{array}\right]
$$

$$
\begin{aligned}
\text { and } & r, j \\
B_{i, j} & =-\sum_{k=i+1}^{j-1} B_{i, k} A_{s-j, s-k}^{t}-A_{s-j, s-i}^{t}, \text { for } 0 \leq i<j \leq s, \\
\text { and } U_{j} & =-\sum_{k=1}^{j-1} U_{k} A_{s-j-1, s-k-1}^{t}-2^{j} S_{s-j}^{t}, \text { for } 1 \leq j \leq s-1 .
\end{aligned}
$$

Proof. It is straightforward to check that $H_{s} G_{s}^{t}=0$. Let $\mathscr{C}$

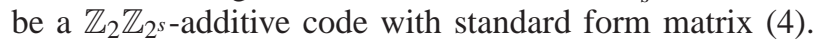
Then $\chi(\mathscr{C})$ has the generator matrix of the form;

$$
\tilde{G}_{S}=\left[\begin{array}{lll}
2^{s-1} \bar{A} & T \\
2^{s-1} S & A
\end{array}\right]
$$

Since $\chi(\mathscr{C})$ is a code over $\mathbb{Z}_{2 s}$ we know from [3] that the matrix in (8) is permutation equivalent to the matrix $\tilde{G}$, by moving the first $k_{0}$ rows to the last row and next moving the first $\alpha$ columns between the last $s-1$ and $s$ blocks;

$\tilde{G}=\left[\begin{array}{cccccccc}I_{k_{1}} & \tilde{A}_{01} & \tilde{A}_{02} & \tilde{A}_{03} & \cdots & \tilde{A}_{0, s-2} & \tilde{A}_{0, s-1} & \tilde{A}_{0, s} \\ 0 & 2 I_{k_{2}} & 2 \tilde{A}_{12} & 2 \tilde{A}_{13} & \cdots & 2 \tilde{A}_{1, s-2} & 2 \tilde{A}_{1, s-1} & 2 \tilde{A}_{1, s} \\ \vdots & \vdots & \vdots & \vdots & \vdots & \vdots & \vdots & \vdots \\ 0 & 0 & 0 & 0 & \cdots & 2^{s-2} I_{k_{s-1}} & 2^{s-2} \tilde{A}_{s-2, s-1} & 2^{s-2} \tilde{A}_{s-2, s} \\ 0 & 0 & 0 & 0 & \cdots & 0 & 2^{s-1} \tilde{I}_{k_{s}} & 2^{s-1} \tilde{A}_{s-1, s}\end{array}\right]$

where, for $0 \leq i \leq s-3,1 \leq j \leq s-2, \tilde{A}_{i, j}=A_{i, j}$ and

$$
\tilde{I}_{k_{s}}=\left[\begin{array}{cc}
I_{k_{s}} & 0 \\
0 & I_{k_{0}}
\end{array}\right], \tilde{A}_{s-1, s}=\left[\begin{array}{cc}
0 & A_{s-1, s} \\
\bar{A}_{01} & T_{0, s}
\end{array}\right]
$$

and for $0 \leq i \leq s-2$,

$$
\tilde{A}_{i, s-1}=\left[A_{i, s-1} 0\right], \tilde{A}_{i, s}=\left[2^{s-i-1} S_{i+1} A_{i, s}\right] .
$$

Again from [3] the generator matrix of the dual code $\chi(\mathscr{C})^{\perp}$ is permutation equivalent to the matrix of the form;

$\tilde{H}=\left[\begin{array}{cccccc}\tilde{B}_{0, s} & \tilde{B}_{0, s-1} & \cdots & \tilde{B}_{0,2} & \tilde{B}_{0,1} & I_{\alpha+\beta-k(\tilde{G})} \\ 2 \tilde{B}_{1, s} & 2 \tilde{B}_{1, s-1} & \cdots & 2 \tilde{B}_{1,2} & 2 \tilde{I}_{k_{s}(\tilde{G})} & 0 \\ \vdots & \vdots & \vdots & \vdots & \vdots & \vdots \\ 2^{s-1} \tilde{B}_{s-1, s} & 2^{s-1} I_{k_{2}(\tilde{G})} & \cdots & 0 & 0 & 0\end{array}\right]$ 
where

$$
\tilde{B}_{i, j}=-\sum_{k=i+1}^{j-1} \tilde{B}_{i, k} \tilde{A}_{s-j, s-k}^{t}-\tilde{A}_{s-j, s-i}^{t} \text { for } 0 \leq i<j \leq s .
$$

We can also write this matrix in the form;

$\tilde{H}=\left[\begin{array}{ccccccc}\tilde{B}_{0, s} & \tilde{B}_{0, s-1} & \cdots & \tilde{B}_{0,2} & \tilde{B}_{0,1} & I_{\alpha-k_{0}} & 0 \\ 2 \tilde{B}_{1, s} & 2 \tilde{B}_{1, s-1} & \cdots & 2 \tilde{B}_{1,2} & 2 \tilde{I}_{k_{s}(\tilde{G})} & 0 & I_{\beta-k(A)} \\ \vdots & \vdots & \vdots & \vdots & \vdots & \vdots & \vdots \\ 2^{s-1} \tilde{B}_{s-1, s} & 2^{s-1} I_{k_{2}(\tilde{G})} & \cdots & 0 & 0 & 0 & 0\end{array}\right]$.

Therefore, by reversing only the column permutation applied before, we can write the generator matrix of the dual code $\chi(\mathscr{C})^{\perp}$ as follows:

$$
\tilde{H}_{s}=\left[\begin{array}{ccc}
-\bar{A}_{01}^{t} I_{\alpha-k_{0}} & U \\
\tilde{T} & 0 & B \\
2 I_{k_{0}} & 0 & 0
\end{array}\right]
$$

where

$$
\begin{gathered}
B=\left[\begin{array}{cccccc}
B_{0, s} & B_{0, s-1} & \cdots & B_{0,2} & B_{0,1} & I_{\beta-k(A)} \\
2 B_{1, s} & 2 B_{1, s-1} & \cdots & 2 B_{1,2} & 2 I_{k_{s}(A)} & 0 \\
\vdots & \vdots & \vdots & \vdots & \vdots & \vdots \\
2^{s-1} B_{s-1, s} & 2^{s-1} I_{k_{2}(A)} & \cdots & 0 & 0 & 0
\end{array}\right], \\
U=\left[\begin{array}{lllll}
U_{s-1} & U_{s-2} & \cdots & U_{1} & 0
\end{array}\right], \tilde{T}=\left[\begin{array}{c}
-T_{0, s}^{t} \\
0 \\
\vdots \\
0
\end{array}\right]
\end{gathered}
$$

where

$B_{i, j}=-\sum_{k=i+1}^{j-1} B_{i, k} A_{s-j, s-k}^{t}-A_{s-j, s-i}^{t}, \quad$ for $0 \leq i<j \leq s$,

and

$$
U_{j}=-\sum_{k=1}^{j-1} U_{k} A_{s-j-1, s-k-1}^{t}-2^{j} S_{s-j}^{t}, \text { for } 1 \leq j \leq s-1 .
$$

Finally, by Proposition 4.3, $\psi\left(\tilde{H}_{S}\right)=H_{S}$ is the generator matrix of $\mathscr{C}^{\perp}$.

Next, we give a corollary that gives the results obtained in [2] and also helps illustrating Theorem 5.1.

Corollary 5.2. Let $\mathscr{C}_{1}$ be a $\mathbb{Z}_{2} \mathbb{Z}_{4}$-additive code of type $\left(\alpha, \beta ; k_{0}, k_{1}, k_{2}\right)$ with standard form matrix (5). Then the parity-check matrix of $\mathscr{C}_{1}$ is permutation equivalent to a matrix of the form;

$$
\left[\begin{array}{ccccc}
-\bar{A}_{01}^{t} & I_{\alpha-k_{0}} & -2 S_{1}^{t} & 0 & 0 \\
-T_{02}^{t} & 0 & -A_{02}^{t}+A_{12}^{t} A_{01}^{t} & -A_{12}^{t} & I_{\beta-k_{1}-k_{2}} \\
0 & 0 & -2 A_{01}^{t} & 2 I_{k_{2}} & 0
\end{array}\right]
$$

Proof. Let $\mathscr{C}_{1}$ be a $\mathbb{Z}_{2} \mathbb{Z}_{4}$-additive code of type $\left(\alpha, \beta ; k_{0}, k_{1}, k_{2}\right)$. Then the generator matrix of $\chi\left(\mathscr{C}_{1}\right)$ is permutation equivalent to a matrix of the form;

$$
\left[\begin{array}{ccccc}
I_{k_{1}} & A_{01} & 0 & 2 S_{1} & A_{02} \\
0 & 2 I_{k_{2}} & 0 & 0 & 2 A_{12} \\
0 & 0 & 2 I_{k_{0}} & 2 \bar{A}_{01} & 2 T_{02}
\end{array}\right]
$$

Therefore, we have the generator matrix of $\chi\left(\mathscr{C}_{1}\right)^{\perp}$ as;

$$
\left[\begin{array}{ccccc}
-2 S_{1}^{t} & 0 & -\bar{A}_{01}^{t} & I_{\alpha-k_{0}} & 0 \\
-A_{02}^{t}+A_{12}^{t} A_{01}^{t} & -A_{12}^{t} & -T_{02}^{t} & 0 & I_{\beta-k_{1}-k_{2}} \\
-2 A_{01}^{t} & 2 I_{k_{2}} & 0 & 0 & 0 \\
0 & 0 & 2 I_{k_{0}} & 0 & 0
\end{array}\right]
$$

After reversing only the column permutation, we can write this matrix in the form of;

$$
\tilde{H}_{s}=\left[\begin{array}{ccccc}
-\bar{A}_{01}^{t} & I_{\alpha-k_{0}} & -2 S_{1}^{t} & 0 & 0 \\
-T_{02}^{t} & 0 & -A_{02}^{t}+A_{12}^{t} A_{01}^{t} & -A_{12}^{t} & I_{\beta-k_{1}-k_{2}} \\
0 & 0 & -2 A_{01}^{t} & 2 I_{k_{2}} & 0 \\
2 I_{k_{0}} & 0 & 0 & 0 & 0
\end{array}\right]
$$

Finally, applying $\psi$ to $\tilde{H}_{s}$ we have the generator matrix of $\mathscr{C}_{1}^{\perp}$ as follows:

$H_{s}=\psi\left(\tilde{H}_{S}\right)=\left[\begin{array}{ccccc}-\bar{A}_{01}^{t} & I_{\alpha-k_{0}} & -2 S_{1}^{t} & 0 & 0 \\ -T_{02}^{t} & 0 & -A_{02}^{t}+A_{12}^{t} A_{01}^{t} & -A_{12}^{t} & I_{\beta-k_{1}-k_{2}} \\ 0 & 0 & -2 A_{01}^{t} & 2 I_{k_{2}} & 0\end{array}\right]$.

Again, note that this matrix is permutation equivalent to the matrix of the form (2). In the sequel, we present two examples that further illustrates Theorem 5.1.

Example 5.3. Let $\mathscr{C}_{2}$ be a $\mathbb{Z}_{2} \mathbb{Z}_{8}$-additive code of type $\left(\alpha, \beta ; k_{0}, k_{1}, k_{2}, k_{3}\right)$ with standard form matrix (6). We can write the parity-check matrix of $\mathscr{C}_{2}$ as follows:

$$
\left[\begin{array}{ccccc}
-\bar{A}_{01}^{t} & I_{\alpha-k_{0}} & -2 S_{2}^{t} & 0 & 0 \\
-T_{03}^{t} & 0 & P-A_{13}^{t}+A_{23}^{t} A_{12}^{t} & -A_{23}^{t} & I_{\beta-k_{1}-k_{2}-k_{3}} \\
0 & 0 & -2 A_{12}^{t} & 2 I_{k_{3}} & 0 \\
0 & 0 & 4 I_{k_{2}} & 0 & 0
\end{array}\right]
$$

where

$$
P=\left[\begin{array}{c}
-4 S_{1}^{t}+2 S_{2}^{t} A_{01}^{t} \\
-A_{03}^{t}+A_{13}^{t} A_{01}^{t}+A_{23}^{t} A_{02}^{t}-A_{23}^{t} A_{12}^{t} A_{01}^{t} \\
-2 A_{02}^{t}+2 A_{12}^{t} A_{01}^{t} \\
-4 A_{01}^{t}
\end{array}\right]
$$

Example 5.4. Let $\mathscr{C}_{3}$ be a $\mathbb{Z}_{2} \mathbb{Z}_{8}$-additive code of type $(3,3 ; 1,3,0,0)$ with standard form matrix,

$$
\left[\begin{array}{llllll}
1 & 1 & 0 & 0 & 0 & 0 \\
0 & 0 & 1 & 1 & 0 & 0 \\
0 & 1 & 1 & 0 & 1 & 0 \\
0 & 1 & 1 & 0 & 0 & 1
\end{array}\right]
$$


Then the additive dual code $\mathscr{C}_{3}^{\perp}$ has generator matrix of the form;

$$
\left[\begin{array}{llllll}
1 & 1 & 0 & 0 & 4 & 4 \\
0 & 0 & 1 & 4 & 4 & 4
\end{array}\right]
$$

\section{Bounds on the minimum distance of $\mathbb{Z}_{2} \mathbb{Z}_{2^{s}}$-additive codes}

In this section we give two bounds for $\mathbb{Z}_{2} \mathbb{Z}_{2^{s}}$-additive codes and compare them.

Since the Gray map defined in [4] is an isometry, the map $\Phi$ defined in (3) transforms Lee distances in $\mathbb{Z}_{2}^{\alpha} \times \mathbb{Z}_{2^{s}}^{\beta}$ to Hamming distances in $\mathbb{Z}_{2}^{n}$, where $n=\alpha+2^{s-1} \beta$. In [1], the weight of any vector in $\mathbb{Z}_{2}^{\alpha} \times \mathbb{Z}_{4}^{\beta}$ was defined. Now, denote the Hamming weight of $v_{1} \in \mathbb{Z}_{2}^{\alpha}$ by $w t_{H}\left(v_{1}\right)$ and denote the Lee weight of $v_{2} \in \mathbb{Z}_{2^{s}}^{\beta}$ by $w t_{L}\left(v_{2}\right)$. We define the weight of $v=\left(v_{1}, v_{2}\right) \in \mathbb{Z}_{2}^{\alpha} \times \mathbb{Z}_{2^{s}}^{\beta}$ as $w t(v)=w t_{H}\left(v_{1}\right)+$ $w t_{L}\left(v_{2}\right)$. We can also define the distance between any two vectors $u, v \in \mathbb{Z}_{2}^{\alpha} \times \mathbb{Z}_{2^{s}}^{\beta}$ as $d(u, v)=w t(u-v)$. Now, we denote the minimum distance between codewords in $\mathscr{C}$ by $d(\mathscr{C})$.

The usual Singleton bound for codes over an alphabet of size $q$ is given in [10] by $d(\mathscr{C}) \leq n-\log _{q}|\mathscr{C}|+1$.

This bound is a combinatorial bound and does not rely on the algebraic structure of the code. In [7] the following Singleton bound for the Lee weight of a quaternary linear code is given. By using this bound we have

$$
\left\lfloor\frac{d(\mathscr{C})-1}{2}\right\rfloor \leq n-\delta-\frac{\gamma}{2}
$$

for a code $\mathscr{C}$ of type $2^{\gamma} 4^{\delta}$.

Theorem 6.1. Let $\mathscr{C}$ be a $\mathbb{Z}_{2} \mathbb{Z}_{2^{s}}$-additive code of type $\left(\alpha, \beta ; k_{0}, k_{1}, k_{2} \ldots, k_{s}\right)$. Then,

$$
\frac{d(\mathscr{C})-1}{2^{s-1}} \leq \frac{\alpha}{2^{s-1}}+\beta-\frac{\left[k_{0}+s k_{1}+(s-1) k_{2}+\ldots+k_{s}\right]}{2^{s-1}}
$$

and

$$
\left\lfloor\frac{d(\mathscr{C})-1}{2^{s-1}}\right\rfloor \leq \alpha+\beta-\left(k_{0}+k_{1}+k_{2}+\ldots+k_{s}\right) .
$$

Proof. We can obtain the bound in (9) by simply applying the Singleton bound given in [10] to $\Phi(\mathscr{C})=C$ where $\Phi$ is defined in (3). Since

$d(\mathscr{C}) \leq n-\log _{q}|\mathscr{C}|+1$ we get

$d(\mathscr{C}) \leq \alpha+2^{s-1} \beta-\left[k_{0}+s k_{1}+(s-1) k_{2}+\ldots+k_{s}\right]+1$.

Therefore, we have

$$
\frac{d(\mathscr{C})-1}{2^{s-1}} \leq \frac{\alpha}{2^{s-1}}+\beta-\frac{\left[k_{0}+s k_{1}+(s-1) k_{2}+\ldots+k_{s}\right]}{2^{s-1}} .
$$

Let $\left(\chi, I_{d}\right): \mathbb{Z}_{2}^{\alpha} \times \mathbb{Z}_{2^{s}}^{\beta} \longrightarrow \mathbb{Z}_{2^{s}}^{\alpha+\beta}$ be the usual inclusion from $\mathbb{Z}_{2}$ to $\mathbb{Z}_{2^{s}}$ that we defined before. Then, it is clear that $d(\mathscr{C}) \leq d(\chi(\mathscr{C}))$.

The bound in (10) can be obtained by using this result and Theorem 4.3 in [7] that is;

$$
\left\lfloor\frac{d(\mathscr{C})-1}{2^{s-1}}\right\rfloor \leq n-\operatorname{rank}(\mathscr{C}) .
$$

Hence we have

$$
\left\lfloor\frac{d(\mathscr{C})-1}{2^{s-1}}\right\rfloor \leq \alpha+\beta-\left(k_{0}+k_{1}+k_{2}+\ldots+k_{s}\right) .
$$

Lemma 6.2. Let $\mathscr{C}$ be a $\mathbb{Z}_{2} \mathbb{Z}_{2^{s}}$-additive code of type $\left(\alpha, \beta ; k_{0}, k_{1}, k_{2}, \ldots, k_{s}\right)$, then the bound in (9) is strictly stronger than the bound in (10) if and only if;

i) $d(\mathscr{C})=2^{s-1} a+1$ and

$k_{0}+k_{s}+\frac{2^{s-1}\left(k_{1}+\ldots+k_{s-1}\right)-\left[s k_{1}+\ldots+2 k_{s-1}\right]}{2^{s-1}-1}<\alpha$.

ii) $d(\mathscr{C})=2^{s-1} a$ and

$k_{0}+k_{s}+\frac{2^{s-1}\left(k_{1}+\ldots+k_{s-1}\right)-\left[s k_{1}+\ldots+2 k_{s-1}\right]}{2^{s-1}-1} \leq \alpha$.

iii) $d(\mathscr{C})=2^{s-1} a+b$ and

$k_{0}+k_{s}+\frac{2^{s-1}\left(k_{1}+\ldots+k_{s-1}\right)-\left[s k_{1}+\ldots+2 k_{s-1}\right]}{2^{s-1}-1}<\alpha+\frac{b-1}{2^{s-1}-1}$

where $1<a \in \mathbb{Z}$ and $1<b<2^{s-1}, b \in \mathbb{Z}$.

Proof. i) If $d(\mathscr{C})=2^{s-1} a+1$ then the bound in (9) is strictly stronger than the bound in (10) if and only if;

$$
\begin{array}{r}
\alpha+2^{s-1} \beta-\left[k_{0}+s k_{1}+\ldots+k_{s}\right]+1 \\
<2^{s-1} \alpha+2^{s-1} \beta-2^{s-1}\left(k_{0}+\ldots+k_{s}\right)+1 \\
\left(k_{0}+k_{s}\right)\left(2^{s-1}-1\right)+2^{s-1}\left(k_{1}+\ldots+k_{s-1}\right)-\left[s k_{1}+\ldots+2 k_{s-1}\right] \\
<\left(2^{s-1}-1\right) \alpha \\
k_{0}+k_{s}+\frac{2^{s-1}\left(k_{1}+\ldots+k_{s-1}\right)-\left[s k_{1}+\ldots+2 k_{s-1}\right]}{2^{s-1}-1}<\alpha .
\end{array}
$$

ii) If $d(\mathscr{C})=2^{s-1} a$ then bound in (9) is strictly stronger than bound in (10) if and only if;

$$
\begin{aligned}
& \alpha+2^{s-1} \beta-\left[k_{0}+s k_{1}+\ldots+k_{s}\right]+1 \\
& <2^{s-1}\left[\alpha+\beta-\left(k_{0}+\ldots+k_{s}\right)+1\right] \\
& \quad\left(k_{0}+k_{s}\right)\left(2^{s-1}-1\right)+2^{s-1}\left(k_{1}+\ldots+k_{s-1}\right) \\
& \quad-\left[s k_{1}+\ldots+2 k_{s-1}\right]<\left(2^{s-1}-1\right)(\alpha+1)
\end{aligned}
$$


$k_{0}+k_{s}+\frac{2^{s-1}\left(k_{1}+\ldots+k_{s-1}\right)-\left[s k_{1}+\ldots+2 k_{s-1}\right]}{2^{s-1}-1}<\alpha+1$,

$k_{0}+k_{s}+\frac{2^{s-1}\left(k_{1}+\ldots+k_{s-1}\right)-\left[s k_{1}+\ldots+2 k_{s-1}\right]}{2^{s-1}-1} \leq \alpha$.

iii) If $d(\mathscr{C})=2^{s-1} a+b$ then bound in (9) is strictly stronger than bound in (10) if and only if;

$$
\begin{aligned}
& \alpha+2^{s-1} \beta-\left[k_{0}+s k_{1}+\ldots+k_{s}\right]+1 \\
& <2^{s-1}\left[\alpha+\beta-\left(k_{0}+\ldots+k_{s}\right)\right]+b, \\
& k_{0}+k_{s}+\frac{2^{s-1}\left(k_{1}+\ldots+k_{s-1}\right)-\left[s k_{1}+\ldots+2 k_{s-1}\right]}{2^{s-1}-1} \\
& <\alpha+\frac{b-1}{2^{s-1}-1} .
\end{aligned}
$$

Now, we give two examples such that, the first one attains the bound in (9) and the second one attains the bound in (10). This shows that these bounds are the best possible ones.

Example 6.3. Let $\mathscr{C}_{4}$ be a $\mathbb{Z}_{2} \mathbb{Z}_{16}$-additive code of type $(1,1 ; 1,0,0,0,0)$ with generator matrix $G_{4}=\left[\begin{array}{ll}1 & 8\end{array}\right]$. Therefore, $s=4, d(\mathscr{C})=9$. Applying the bound in (9) we have;

$$
\begin{aligned}
\frac{d(\mathscr{C})-1}{8} & \leq \frac{\alpha}{8}+\beta-\frac{k_{0}+4 k_{1}+3 k_{2}+2 k_{3}+k_{4}}{8} \\
& \Rightarrow \frac{9-1}{8} \leq \frac{1}{8}+1-\frac{1}{8} \\
1 & \leq 1 .
\end{aligned}
$$

Example 6.4. Consider a $\mathbb{Z}_{2} \mathbb{Z}_{8}$-additive code $\mathscr{C}_{5}$ generated by the following generator matrix

$$
G_{5}=\left[\begin{array}{llll}
1 & 0 & 0 & 4 \\
0 & 4 & 0 & 4 \\
0 & 0 & 4 & 4
\end{array}\right]
$$

So, $\mathscr{C}_{5}$ is of type $(1,3 ; 1,0,0,2)$ and

$$
\begin{array}{r}
\mathscr{C}=\{(0000),(1004),(0404),(0044),(1400), \\
(1040),(0440),(1444)\} .
\end{array}
$$

Then $d(\mathscr{C})=5$. If we apply bound in (10) we get;

$$
\begin{aligned}
\left\lfloor\frac{d(\mathscr{C})-1}{4}\right\rfloor & \leq \alpha+\beta-\left(k_{0}+k_{1}+k_{2}+k_{3}\right) \\
\left.\mid \frac{5-1}{4}\right\rfloor & \leq 1+3-(1+0+0+2) \\
1 & \leq 1 .
\end{aligned}
$$

\section{Conclusion}

This paper presents a generalization of $\mathbb{Z}_{2} \mathbb{Z}_{4}$ additive codes which has been introduced lately. The paper studies the structure of $\mathbb{Z}_{2} \mathbb{Z}_{2^{s}}$ additive codes. An inner product is introduced and by introducing the dual concept of an additive code, the structure of the duals of additive codes are studied. Some bounds that are attained by this family of codes are introduced. Since this a new topic and a new direction, the classical questions and problems that are solved for linear codes await the research community for this particular family.

\section{Acknowledgement}

The authors wish to thank the anonymous referees for their valuable remark that helped us to improve this paper.

\section{References}

[1] M. Bilal, J. Borges, S. T. Dougherty, C. FernándezCórdoba, "Optimal Codes Over $\mathbb{Z}_{2} \times \mathbb{Z}_{4}$ ", VII Jornadas de Matemática Discreta y Algorítmica Castro Urdiales, 7-9 de julio de (2010).

[2] J. Borges, C. Fernández-Córdoba, J. Pujol, J. Rif́a and M. Villanueva, " $\mathbb{Z}_{2} \mathbb{Z}_{4}$-linearcodes: Generator Matrices and Duality”, Designs, Codes and Cryptography, 54, 167-179 (2010).

[3] A. R. Calderbank and N. J. A. Sloane, "Modular and p-adic Codes", Designs, Codes and Cryptography, 6, 21-35 (1995).

[4] C. Carlet, “ $\mathbb{Z}_{2^{k}}$-linear Codes", IEEE Trans. Inform. Theory, 44, 1543-1547 (1998).

[5] P. Delsarte, "An Algebraic Approach to the Association Schemes of Coding Theory", Philips Research Rep. Supp., 10, (1973).

[6] P. Delsarte, V. Levenshtein, "Association Schemes and Coding Theory", IEEE Trans. Inform. Theory, 44, 24772504 (1998).

[7] S. T. Dougherty and K. Shiromoto, "Maximum Distance Codes Over Rings of Order 4", IEEE Trans. Inform. Theory, 47, 400-404 (2001).

[8] A. R. Hammons, V. Kumar, A. R. Calderbank, N.J.A. Sloane, P. Solé, "The $\mathbb{Z}_{4}$-linearity of Kerdock, Preparata, Goethals, and Related Codes", IEEE Trans. Inform. Theory, 40, 301-319 (1994).

[9] J. Pujol, J. Rifá, “Translation Invariant Propelinear Codes”, IEEE Trans. Inform. Theory, 43, 590-598 (1997).

[10] R. C. Singleton, "Maximum Distance q-ary Codes", IEEE Trans. Inform. Theory, 10, 116 -118 (1964). 


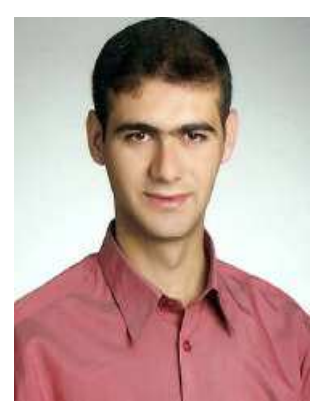

Ismail Aydogdu is

a PhD student and a research assistant in Department of Mathematics at Yildiz Technical University, Istanbul. $\mathrm{He}$ is preparing his $\mathrm{PhD}$ thesis about additive codes. His main research interests are Algebraic Coding Theory and Information Theory.



Irfan Siap is professor in Department of Mathematics at Yildiz Technical University, Istanbul. $\mathrm{He}$ is also the head of the same department. $\mathrm{He}$ received his $\mathrm{PhD}$ degree in Mathematics from The Ohio State University in The United States. His research interest is mainly in the area of Algebra and Its Applications, especially Algebraic Coding Theory, Cryptography, Information Theory, Discrete Mathematics, Combinatorics, Cellular Automata, Fuzzy Coding and Mathematics Education. He has published many research articles in reputed international journals of mathematical and engineering sciences. He is one of the Associate Editors (2007-) of The Franklin Institute Journal among some others. He has been serving as a referee for many well distinguished journals. 